\title{
Inversión en actividades de ciencia, tecnología e innovación: el caso de Colombia y Ecuador *
}

\author{
Investment in Science, Technology, and Innovation Activities: The Case of \\ Colombia and Ecuador \\ Luisa Fernanda Echeverría-King \\ Doctorando en Educación, Servicio Nacional de Aprendizaje, \\ Bogotá-Colombia, lecheverriaking@gmail.com \\ Jorge Pinto \\ Magíster en Gestión y Política Pública, Agencia de Cooperación Internacional de Corea del Sur, \\ Quito-Ecuador, jorgeandrespintol@hotmail.com \\ María Ángela Lorena Mosquera-Montoya \\ Magíster en Análisis en Problemas Políticos, Económicos e Internacionales Contemporáneos, \\ Escuela de Postgrados de Policía, Bogotá-Colombia, lorena.mosquera@espoledu.co
}

\begin{abstract}
Cómo citar / How to cite
Echeverría-King, L. F.; Pinto, J.; Mosquera-Montoya, M. A. L. (2021). Inversión en actividades de Ciencia, Tecnología e Innovación: el caso de Colombia y Ecuador. Revista CEA, v. 7, n. 14, e1672.

https://doi.org/10.22430/24223182.1672
\end{abstract}

Recibido: 22 de noviembre de 2020

Aceptado: 12 de marzo de 2021

\section{Resumen}

Las inversiones en actividades de ciencia, tecnología e innovación son indicadores de desarrollo y competitividad para los países. La presente investigación pretende describir y analizar las inversiones en actividades de ciencia, tecnología e innovación realizadas por Colombia y Ecuador entre 2010 y 2014, años marcados por la bonanza petrolera. Para ello se realizó una metodología de corte cuantitativa y exploratoria, por medio de una revisión documental y sistemática de informes oficiales, Durante el periodo estudiado se realizaron inversiones en maestría y doctorado para la formación de personal, especialmente en Ecuador, mientras que en Colombia la producción bibliográfica fue en aumento. Por otro lado, en Colombia solo se registraron un total de 806 patentes, frente a 2709 registradas del vecino país. Sin embargo, a Colombia se le concedieron un total de 2674 patentes en diseños industriales, mientras que, a Ecuador, solo le fueron concedidos 764. Asimismo, se identificó que la inversión privada en las actividades de ciencia, tecnología e innovación aún es baja y que se

\footnotetext{
* Este artículo se deriva del proyecto titulado «Inversión en actividades de ciencia, tecnología e innovación: el caso de Colombia y Ecuador» y ha sido financiado con recursos propios.
} 
pueden fortalecer también las inversiones provenientes de otros sectores. Estos estudios ayudan a visibilizar los enfoques de las inversiones realizadas, y representan una oportunidad para observar cómo los países han concentrado sus esfuerzos hacia la generación de nuevo conocimiento. Se hacen necesarios estudios de impacto para evaluar cómo estas inversiones han apoyado a los países a ser más competitivos y a crear nuevos modelos económicos.

Palabras clave: política pública, ciencia tecnología e innovación, inversión pública, desarrollo económico, registro de patentes.

Clasificación JEL: E60, E61, H52, O30, O31, O32, O38.

\title{
Highlights
}

- La inversión en Colombia en actividades de ciencia, tecnología e innovación (ACTI) impactó en las publicaciones y en la formación académica de alto nivel.

- Para Ecuador, la inversión en actividades de ciencia, tecnología e innovación (ACTI) impactó la formación de alto nivel y en el registro de patentes.

- La inversión del sector productivo se ha evidenciado como un reto para la ciencia, la tecnología y la innovación (CTI).

- Es necesario generar estrategias e inversiones a largo plazo para la ciencia, tecnología e innovación (CTI).

\begin{abstract}
Investments in science, technology, and innovation activities are indicators of the development and competitiveness of countries. This study aims to describe and analyze the investments in science, technology, and innovation activities made by the governments of Colombia and Ecuador between 2010 and 2014, a period marked by the oil bonanza. For that purpose, we employed a quantitative and exploratory methodology that included a systematic review of official reports. During the period under study, both nations invested in master's and doctoral training, especially Ecuador, and Colombia experienced an increase in its bibliographic production. Colombia obtained 806 patents, while Ecuador registered 2,709. However, Colombia registered 2,674 industrial designs; and Ecuador, only 764. It was found that private investments in science, technology, and innovation activities are still low, and investment from other sectors can be improved. This type of study helps to reveal the approaches of investments that have been made and offers an opportunity to observe the way countries have focused their efforts on the generation of new knowledge. Future research should include impact assessments to evaluate the way these investments have helped countries to be more competitive and create new economic models.
\end{abstract}

Keywords: Public policy, science technology and innovation, public investment, economic development, patent registration.

JEL classification: E60, E61, H52, O30, O31, O32, O38.

\section{Highlights}


- Colombia's investment in science, technology, and innovation activities (STIA) had an impact on its number of publications and higher education.

- Ecuador's investment in STIA had an impact on its higher education and patent registration.

- Productive sector investments are a challenge for STI.

- National governments should make long-term investments in STI.

\section{INTRODUCCIÓN}

La Ciencia, la tecnología y la innovación (CTI) es un tema de relevancia para las naciones en el mundial (Lemus Delgado, 2020; Lee et al., 2018), ya que evidencia que, a mayor inversión en este sector, mayor incremento de riqueza en las naciones, así como de su producto interno bruto (PIB) per cápita (Chaves et al., 2020). La inversión en actividades de ciencia, tecnología e innovación (ACTI) no sólo mejora el desarrollo económico de los países, sino que, además, es un factor fundamental para su crecimiento (Wu et al., 2019; Cheng \& Wang, 2019). Por tal razón, a partir de la Segunda Guerra Mundial se hace necesaria la creación de políticas públicas encaminadas a mejorar la aplicación de la CTI en el mundo (Albert \& Laberge, 2007), rol asumido por los que hoy se denominan países industrializados, según la Organización de las Naciones Unidas para la Educación, la Ciencia y la Cultura (UNESCO, 2016), los cuales destinan más del 1.5\% del PIB en CTI. Este proceso fue liderado por Estados Unidos, y quedó demostrado en 1945 en el informe "Ciencia: la frontera sin fin», de Vannevar Bush, citado por Loray (2017), por el cual se crearon las primeras instituciones de CTI, vislumbrando el impacto de esta temática en el ámbito internacional.

El proceso de globalización, y el creciente auge de las sociedades de conocimiento (Etokakpan et al., 2020), hace «innegable que para que los países puedan avanzar por una senda de crecimiento sostenido, resulta indispensable y necesario impulsar, a nivel nacional y regional, las actividades relacionadas con la CTI» (Perfetti, 2010). No obstante,

el desarrollo en políticas de CTI en América Latina ha sido tardío y no obedece, en principio, a iniciativas propias de los países de la región, son producto de mirar al norte (Europa y Estados Unidos) como los referentes, como los agentes de desarrollo a imitar. (Giraldo Gutierrez et al., 2020 p. 295)

Esto ha generado que en esta región la inversión destinada a la ciencia, la tecnología e la innovación no cumpla con los estándares internacionales, que presente grandes variaciones y fluctuaciones en el tiempo, que sea débil, sin continuidad y desequilibrada. Giraldo Gutiérrez et al., (2020) exponen que esto se debe a que no ha habido un accionar efectivo y eficiente en la región en materia de CTI y que los picos de caída de inversión sean una constante en América Latina.

En este sentido, surge la inquietud de revisar cómo se ha direccionado la inversión en CTI en países latinoamericanos como Ecuador y Colombia, los cuales comparten una historia similar, pues son países fronterizos y pertenecen a la Comunidad Andina de Naciones (CAN), marcados por la bonanza petrolera y económica, lo que incentivó la inversión gubernamental en las actividades de ciencia, tecnología e innovación (ACTI). Esto permite tener un conocimiento más profundo sobre la destinación presupuestal que se hace en CTI en estos países en vías de desarrollo y la relevancia que reviste en cada uno de ellos, especialmente en el período propuesto entre 2010 y 2014. 


\section{MARCO TEÓRICO}

\section{La Ciencia, la tecnología y la innovación en América Latina}

América Latina está conformada por un conjunto de países que comparten realidades culturales, políticas y económicas, y a la vez se encuentran marcados por ser una de las regiones más inequitativas del mundo. La Unesco (2015) expone que, desde la década de 1960, las crisis económicas y políticas de la región han tenido un impacto directo en el diseño y desempeño de las políticas de CTI tanto para la oferta como para la demanda, afectando la planeación a largo plazo de políticas públicas en la mayoría de los países debido a esquemas fallidos de gobernanza. Si bien en los años 90 se generaron ajustes importantes a los sistemas de CTI de los países, con nuevas instituciones, marcos legales e incentivos para la investigación y la innovación, aún persisten brechas en la región que no permiten que se avance en CTI, como se observa a continuación (Aguirre-Bastos \& Gupta, 2009). En el caso de América Latina, dado que las políticas referentes a CTI se han caracterizado por ajustarse «a la realidad político-económica de los países de la región, marcada por importantes periodos de incertidumbre e inestabilidad» (Loray, 2017), denota una debilidad en la implementación de estas políticas y consecuentemente en la inversión, que en muchos casos no supera el $1 \%$ del PIB. Asimismo, se observan esfuerzos atomizados en diversos programas y acciones de fomento que no convergen entre sí, por lo cual se requiere crear estrategias que complementen los esfuerzos y creen sinergias. Las prioridades nacionales deberían estar alineadas frente a áreas estratégicas de conocimiento que se requieran para la transformación de los diversos sectores económicos (Maravert Alba et al., 2016).

Según Bortagaray (2016), la inversión en las actividades de ciencia, tecnología e innovación en América Latina históricamente ha provenido del sector público y existe un divorcio entre el sector productivo y la generación conjunta de nuevo conocimiento. Siguiendo a esta autora, se observa que las comunidades científicas son marginales o reducidas, falta la orientación de la ciencia a la solución de problemas y se evidencia una baja productividad en comparación con otras zonas geográficas del mundo. De acuerdo con Casas (2019), los sistemas de ciencia, tecnología e innovación en América Latina se encuentran en tensión por diferentes factores, como lo son las situaciones políticas en la región, los lineamientos implementados por países desarrollados, sugerencias expedidas por organismos internacionales y las percepciones de ciencia, tecnología e innovación en la sociedad. Esto se relaciona, según la autora, con comunidades académicas elitistas en las que participan unos pocos y no se incluyen a gran escala a actores relevantes como los sectores productivos y diversos sectores sociales. Según Velo y Perrotta (2020), en Latinoamérica se ha observado la denominada «integración subordinada» a megaproyectos científicos, donde se evidencia, en gran medida, la dependencia académica, de recursos y de capacidades de países del Norte global. Incluso, Abello et al., (2001), indican que se requieren más recursos para la formación de investigadores, inversiones en CTI por parte de las empresas de la mano con incentivos por parte del Estado, así como programas de fomento a la CTI desde las instituciones de educación superior, con el fin de poder aportar a la sana alineación entre la ciencia, la producción y la competitividad.

Scargiali (2019), por su parte, explica que, partiendo de la década del 2000, las economías latinoamericanas comenzaron nuevos modelos de crecimiento y expansión de cara a mejorar la situación en la región afectada por procesos neoliberales, favoreciendo modelos de integración 
regional y la creación de políticas supra regionales de CTI. Estos modelos permitieron las inversiones en los sistemas de innovación en la región, en especial para el apoyo y desarrollo tecnológico de las pymes, que constituyen importantes actores de los sectores productivos en América Latina (Crespi \& Dutrénit, 2013). Aunque desde la década del 2000 se ha venido trabajando en enfoques sistémicos y más comprehensivos para los sistemas nacionales de investigación e innovación en América Latina, siguiendo en cierta manera los avances evidenciados por países desarrollados, fue solo hasta el año 2013 donde se evidenció que la región aún no contaba con una base científica importante (resultante por la falta de capital humano formado), no existían suficientes empresas interesadas en invertir en investigación y desarrollo (I+D) y se requería un acercamiento mejor enfocado de la ciencia con la sociedad, con el fin de cerrar brechas y problemas existentes en la región (Crespi \& Dutrénit, 2013).

Por su parte, la Unesco (2015) expone que los mecanismos más tradicionales de promoción científica para la investigación en América Latina han sido históricamente las subvenciones y la generación de centros de excelencia, muchos de ellos creados en colaboración internacional. Las subvenciones pueden apuntar a infraestructura y el equipamiento de los laboratorios en forma de subvenciones para viajes y ayudas a la investigación y al desarrollo tecnológico. Durante las últimas dos décadas, la mayoría de los países latinoamericanos creó fondos específicos para la investigación y la innovación competitivos. En 2013, las patentes otorgadas representaron alrededor del 1\% del número concedido a las economías de altos ingresos. Ibañez-Marti (2018), por su parte, indica que las inversiones que se realizan en América Latina en educación científica en edades tempranas, así como en actividades de divulgación y cultura científica, las cuales deberán ir de la mano con inversiones en infraestructura y tecnologías de la información, deben fortalecerse, y son un reto para la región, no solo por la falta de estrategias de fomento, sino también por la inequidad y problemas de acceso a las mismas. Franco Avellaneda y Lingsingen (2011) indican que, para lograr impactar estos dos aspectos, se hace necesario lograr un equilibrio entre la política pública, los actores involucrados y la educación, teniendo en cuenta que la apropiación del conocimiento científico sucede por medio de procesos formativos adaptados a los contextos culturales y a los diversos individuos. En este sentido, uno de los objetivos de la CTI en Latinoamérica deberá ser la formación de masa crítica que valore el conocimiento científico y que pueda desarrollar competencias relacionadas con el pensamiento crítico, la indagación y la innovación (Narro Robles, 2008).

Según un informe publicado en 2020 por parte de la Red de Indicadores de Ciencia y Tecnología Iberoamericana e Interamericana- (RICYT), la inversión en I+D en América Latina representa sólo el $2.8 \%$ del monto invertido en el mundo, aunque es importante resaltar que en el contexto global el crecimiento en la inversión de I+D de América Latina fue significativo hasta el año 2015; a partir de esta fecha, por diversas situaciones políticas y económicas, se evidenciaron altibajos. Otro aspecto para mencionar es la concentración de I+D, pues solo Brasil representa el $64 \%$ de los esfuerzos de la región, seguido de México con un $13 \%$ y Argentina con un $8 \%$. Cabe resaltar que esta brecha está relacionada con el tamaño y relevancia de estas economías en la región latinoamericana. En los años comprendidos para este estudio, la cantidad de artículos publicados en revistas de impacto creció sustancialmente, en especial en países como Colombia, el cual, según la RICYT (2020), duplica su publicación en la base datos Scopus durante los años comprendidos entre 2010 y 2014.

Sin embargo, un incremento de la inversión en CTI deberá ir acompañado con medidas y políticas sociales que permitan que las poblaciones en desventaja o vulnerables puedan aprovechar estas oportunidades y no se generen brechas que amplíen el problema de la desigualdad latinoamericana 
(Pérez Hernández et al., 2018). Asimismo, se deben tener en cuenta proyecciones, inversiones y políticas más a largo plazo, donde la CTI pueda ser un motor de desarrollo de las sociedades, apoyando la equidad social y la democratización del conocimiento, superando de esta forma los esquemas y objetivos cortoplacistas existentes que no permiten avanzar de manera sustancial y coordinada en la materia (García Guzmán, 2011).

\section{El caso de Colombia y Ecuador}

Para Colombia y Ecuador, la construcción de sus políticas públicas en CTI empezó en la década de los sesenta, las cuales tienen un marco legal basado en la Constitución Política de cada uno de estos países, así:

En la Constitución Política de Colombia, Artículo 71 (1991)

La búsqueda del conocimiento y la expresión artística son libres. Los planes de desarrollo económico y social incluirán el fomento a las ciencias y, en general, a la cultura. El Estado creará incentivos para personas e instituciones que desarrollen y fomenten la ciencia y la tecnología y las demás manifestaciones culturales y ofrecerá estímulos especiales a personas e instituciones que ejerzan estas actividades.

De la misma manera, en la Constitución Política del Ecuador en los artículos 277 y 298 (2008) se menciona la importancia de desarrollar actividades en pro de mejorar la ciencia, la tecnología y la innovación en ese país. Además, se establecen partidas presupuestales para incentivar estas áreas en las entidades territoriales. Lo anterior demuestra que la CTI se encuentra incluida dentro de las normas que rigen estos Estados, pero que a pesar de ello su implementación ha sido precaria, como se demostrará en los análisis de inversión en estas áreas realizada por estos países.

Colombia y Ecuador, en su compromiso para entrar a la sociedad del conocimiento, han instaurado, mediante sus políticas, instituciones que controlan la implementación de la CTI, quienes a través de una asignación presupuestal (inversión en I+D) construyen una agenda de ciencia, tecnología e innovación que busca potenciar la producción de nuevo conocimiento. Sin embargo, existe una ausencia importante en los propósitos de implementación, en tanto los elementos conceptuales de la apropiación no son considerados (Giraldo Gutiérrez et al., 2020). En los países objeto de estudio se crearon instituciones tendientes a regular la CTI. Colombia lo hizo a través del Decreto 585 de 1991, mediante el cual se conformó el Instituto Colombiano para el Desarrollo de la Ciencia y la Tecnología; no obstante, a partir de la promulgación de la Ley 1286 de 2009, esta se transformó en el Departamento Administrativo de Ciencia, Tecnología e Innovación (Colciencias), el cual se convirtió en un organismo principal de la CTI en este país y cuyo objetivo no es otro que formular, dirigir, orientar, ejecutar e implementar la política de Estado en la materia, a través de cuatro grandes áreas de trabajo, como lo menciona Giraldo Gutiérrez et al., (2020): educación para la investigación, investigación, innovación y mentalidad y cultura científica.

En este mismo sentido, de conformidad al marco legal ecuatoriano, el Sistema Nacional de Ciencia, Tecnología, Innovación y Saberes Ancestrales se encuentra normado a través del Código Orgánico de la Economía Social de los Conocimientos, Creatividad e Innovación (Asamblea Nacional República de Ecuador, 2016), el cual en su artículo No. 7 señala:

Revista CEA, ISSN-p 2390-0725, ISSN-e 2422-3182, Vol. 7 - No. 14, mayo-agosto 2021, e1672 
La Secretaría de Educación Superior, Ciencia, Tecnología e Innovación (Senescyt) es parte de la Función Ejecutiva, y tiene a su cargo la rectoría de la política pública nacional en las materias regladas por este Código, así como la coordinación entre el sector público, el sector privado, popular y solidario, las instituciones del Sistema de Educación Superior y los demás sistemas, organismos y entidades que integran la economía social de los conocimientos, la creatividad y la innovación. (p. 6)

Este código entró en vigencia, por disposición legislativa, a partir del 9 de diciembre de 2016 y otorga a la Senescyt supracompetencias y responsabilidades que la convierten en la única institución pública autorizada o encargada de dar viabilidad al desarrollo de investigaciones en el país, sobre todo en aquellas áreas consideradas de interés nacional. Estas competencias, si bien fueron ampliadas a partir de la aprobación del mencionado Código, han sido responsabilidad de la Senescyt desde su creación en 1994, convirtiéndola en la entidad que mayor cantidad de dinero destina y canaliza al desarrollo de actividades de ciencia, tecnología e innovación. Para citar un ejemplo, según la Encuesta Nacional sobre las actividades en ciencia, tecnología e innovación (ACTI), realizada en Ecuador en 2014, el gasto en investigación, desarrollo e innovación $(I+D+i)$, con relación al PIB, fue de $0.44 \%$, lo cual equivale a USD 259.81 millones, de los cuales tan solo USD 6.40 millones corresponden a gastos realizados por parte del sector privado (Secretaría de Educación Superior, Ciencia, Tecnología e Innovación, 2014).

\section{METODOLOGÍA}

\section{Objetivos de investigación}

El presente artículo pretende describir y analizar las inversiones en actividades de ciencia, tecnología e innovación realizadas por Colombia y Ecuador, entre los años 2010 y 2014, teniendo en cuenta informes oficiales de entidades de CTI de ambos países como fuentes válidas.

Se ha escogido este periodo por ser un espacio de tiempo marcado por la bonanza petrolera en la región. Según Baquero Méndez y Mieles López (2015), entre 2010 y 2014 el precio del barril de petróleo en Ecuador fue en promedio de 93 dólares, lo que tuvo como consecuencia que el Estado ecuatoriano ganara grandes recursos por las actividades extractivas. Según estos autores, en el año 2014 Ecuador extrajo 203 millones de barriles de petróleo, alcanzando un récord nacional. En el caso de Colombia, según Gómez Londoño (2015), entre los años 2002 y 2011, la producción petrolera se incrementó aproximadamente un 58\%, los precios subieron más del $300 \%$ y los ingresos por ventas un $548 \%$. Entre otras cosas, las exportaciones de crudo alcanzaron el $50 \%$ del total de las ventas al exterior en Colombia, y cerca del $12 \%$ del PIB.

\section{Diseño de la investigación}

El presente estudio es de tipo exploratorio y descriptivo, pues busca mostrar aspectos desconocidos o poco estudiados de la inversión en CTI entre los años 2010 y 2014 en Colombia y Ecuador. Los 
estudios descriptivos tienen la intención de exponer hechos, características y situaciones de forma sistemática y objetiva (Martín Marín, 2011).

\section{Instrumentos}

Para esta investigación se utilizó una técnica de recolección de datos basada en fuentes secundarias (revisión bibliográfica), donde se ejecutó una revisión documental de los informes oficiales generados por Colombia y Ecuador sobre las inversiones en actividades de CTI. Estos informes son generados por los países con base en criterios de manuales internacionales en CTI. En Colombia, estos informes son adelantados por el Observatorio Colombiano de Ciencia y Tecnología (OCyT), mientras que en el Ecuador, hasta el año 2014, eran ejecutadas por la Secretaría de Educación Superior, Ciencia, Tecnología e Innovación (Senescyt).

\section{Procedimiento y análisis de la información}

Para llevar a cabo la revisión documental se generaron indicadores para la abstracción de la información de los informes oficiales:

Porcentaje del PIB invertido en las ACTI.

Porcentaje del PIB ejecutado para acciones de I+D.

Inversión en ACTI por tipo de recurso.

Inversión en ACTI por tipo de actividad.

Después de presentar los datos de manera sistemática por cada indicador, a continuación, se presentan resultados donde se comparan el número de becas y créditos asignados para maestría y doctorado, la producción bibliográfica y los títulos de propiedad industrial obtenidos por los dos países entre los años objeto de estudio (2010-2014). Estos elementos constituyen indicadores de resultados e impacto académico establecidos por el Manual de Frascati y el Manual de Oslo como referentes de medición de la inversión en CTI realizada por los países.

\section{RESULTADOS}

\section{Caso Colombia: inversión en ACTI entre los años 2010-2014}

En este apartado se revisarán las inversiones realizadas por Colombia en esta materia entre 2010 y 2014, teniendo en cuenta las dimensiones establecidas por el Manual de Frascati de la Organización para la Cooperación y el Desarrollo Económicos (OCDE, 2015) expuestas en la Figura 1. 

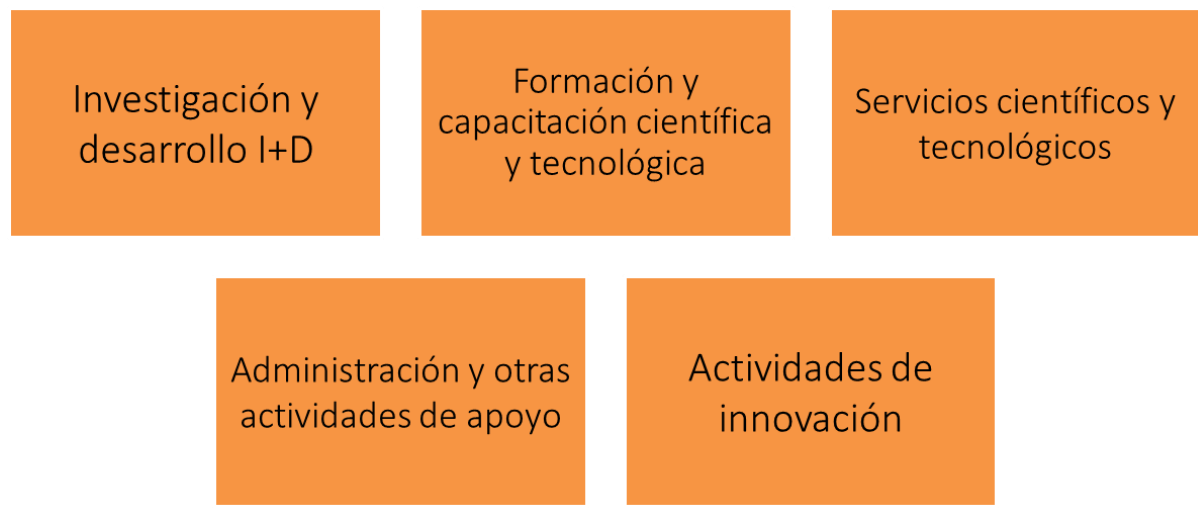

Figura 1. Actividades de ciencia, tecnología e innovación (ACTI)

Figure 1. Science, technology, and innovation activities (STIA) Fuente: elaboración propia a partir de OCDE, 2015.

Al revisar los datos discriminados de las inversiones en ACTI, se observa que para el año 2010 la inversión en estas actividades fue equivalente al 0.497\%, mientras que en 2011 bajó ligeramente a $0.487 \%$ (ver Tabla 1). Igualmente es importante resaltar que el presupuesto asignado a Colciencias en 2010 fue equivalente a 232287 millones de pesos y representó el presupuesto más alto asignado a esa entidad hasta ese momento. Esto significó un incremento del 67\% con respecto al año 2009 (Consejo Privado de Competitividad de Colombia, 2011). La inversión en ACTI, en los años 2012 y 2013, subió ligeramente. Cabe resaltar que, según un informe publicado por el Departamento Administrativo Nacional de Estadística (DANE, 2014), el subsector que más reportó inversión en ACTI en 2012 fue el de educación superior, con un monto de USD 214253000, seguido del subsector de actividades bancarias, con $\$ 107976000$ (estas inversiones fueron reportadas en pesos colombianos; sin embargo, para efectos de este artículo se adaptaron a dólares estadounidenses, teniendo en cuenta la tasa de cambio representativa del mercado [TRM]) de diciembre de 2012 [1 USD=1.768]). En 2013, nuevamente el subsector de educación superior fue el que reportó una mayor inversión en ACTI, con un monto correspondiente a $\$ 278198000$. Correos y telecomunicaciones ocuparon el segundo lugar, con una estimación de \$247189000. Al revisar los datos del año 2014, al cierre del primer gobierno e inicio del segundo periodo del expresidente Juan Manuel Santos, se observó que el incremento en inversión para ACTI continuaba. En el Plan Nacional de Desarrollo (DNP) 2014-2018, se estableció la meta de aumentar la inversión en ACTI hasta el $0.9 \%$ del PIB al finalizar este periodo (Departamento Nacional de Planeación [DNP], 2014). Para 2014, la inversión en ACTI fue equivalente al $0.638 \%$ del PIB.

Tabla 1. Porcentaje del PIB aportado para ACTI y Porcentaje del PIB aportado para I+D 2010-2014 en Colombia Table 1. Percentage of Colombia's GDP devoted to STIA and R\&D from 2010 to 2014

\begin{tabular}{ccc}
\hline Año & Porcentaje PIB-Inversión en ACTI & Porcentaje PIB-Inversión en I+D \\
\hline 2010 & $0.497 \%$ & $0.192 \%$ \\
\hline 2011 & $0.487 \%$ & $0.186 \%$ \\
\hline 2012 & $0.484 \%$ & $0.215 \neg \%$ \\
\hline 2013 & $0.5 \%$ & $0.224-\%$ \\
\hline 2014 & $0.638 \%$ & $0.251 \%$ \\
\hline
\end{tabular}


En cuanto a las acciones enfocadas en investigación y desarrollo, se observa que Colombia invirtió en el año 2010 sólo el 0.192\% de su PIB y en 2011 el 0.186\%; por su parte, para los años 2012 y 2013 hubo un incremento frente al bienio anterior, pasando en 2012 al $0.215 \%$ y en 2013 al $0.224 \%$. Cabe resaltar que en un informe generado por el Consejo Privado de Competitividad de Colombia (CPC) (2013), se expone que la inversión en estas áreas (investigación y desarrollo) por parte del sector privado es aún baja. En cuanto al año 2014, se observa que se incrementó levemente en comparación con el bienio anterior, pasando en el año 2014 al 0.251\% (Lucio et al., 2015). Es preciso destacar que un informe publicado por el DNP y la Dirección de Seguimiento y Evaluación de Políticas PúblicasDsepp (2014), destacó que las actividades de I+D fueron generadas principalmente por instituciones de educación superior (IES) y el Servicio Nacional de Aprendizaje (SENA), seguidamente de empresas y de centros de investigación.

Al revisar los tipos de recursos para la financiación de las ACTI, Salazar et al., (2011) diferencian entre recursos públicos, privados e internacionales. En el año 2010, el $45.97 \%$ de las ACTI fueron financiadas con recursos públicos, mientras que el $50.74 \%$ con recursos privados y un $3.30 \%$ de recursos internacionales. Por su parte, en el año 2011 un 47.61\% de ACTI se ejecutó con recursos públicos, frente a un $49.84 \%$ de recursos privados y un $2.55 \%$ de recursos internacionales. Se observa, en este sentido, que la inversión internacional es muy poca y que la financiación por parte del sector privado podría incrementarse, teniendo en cuenta que son un agente importante en el ecosistema de innovación (ver Tabla 2). En cuanto a los años 2012 y 2013, se muestra un incremento en la inversión pública, pasando en 2012 de 61.26\%, al 63.59\% en 2013. Lo anterior evidenció que tanto los recursos privados como internacionales representaron un porcentaje menor en este bienio que en el anterior. Los recursos privados alcanzaron el $37.12 \%$ en 2012 y el $39.47 \%$ en 2013, mientras que los recursos internacionales representaron el $1.62 \%$ en 2012 y el $1.19 \%$ en 2013 . Es importante destacar lo siguiente: cuando se emitió la Ley 1530 (2012), esta apoyó la organización del Sistema General de Regalías (SGR), lo cual ayudó a una distribución más equitativa de los recursos de CTI en el país (DNP y DSEPP, 2012). En cuanto al año 2014, se reportó que los recursos públicos fueron equivalentes al $54.40 \%$. Al revisar los recursos privados, se observa un leve incremento en la inversión de dichos recursos, reportando en 2014 el 44.25\%. Los recursos internacionales, por su parte, evidenciaron el 1.35\% en 2014 (Lucio et al., 2015).

Tabla 2. Porcentaje del PIB aportado para ACTI y Porcentaje del PIB aportado para I+D 2010-2014 en Colombia Table 2. Sources of investment in STIA in Colombia

\begin{tabular}{cccccc}
\hline Tipo de recurso & 2010 & 2011 & 2012 & 2013 & 2014 \\
\hline Públicos & $45.97-\%$ & $47.61 \%$ & $61.26 \%$ & $63.59 \%$ & $54.40 \%$ \\
\hline Privados & $50.74 \%$ & $49.84 \%$ & $37.12 \%$ & $39.47 \%$ & $44.25 \%$ \\
\hline Internacionales & $3.30 \%$ & $2.55 \%$ & $1.62 \%$ & $1.19 \%$ & $1.35 \%$ \\
\hline \multicolumn{5}{c}{ Fuente: Salazar et al., (2011), Lucio et al., (2013) y Lucio et al., (2015). }
\end{tabular}

Pasando a las inversiones por tipo de actividades (ver Tabla 3), se observa que en I+D se invirtió, en el año 2010, la suma de USD 542352000, y en 2011 se evidencia un ligero incremento hasta USD 559885000 (inversiones reportadas en pesos colombianos que se adaptaron a dólares estadounidenses). En la formación y capacitación científica y tecnológica, la inversión en 2010 alcanzó USD 98137000 y en 2011 aumentó a USD 119743000. Según el Informe de Gestión 2011 (Colciencias, 2011), en ese año se ampliaron los recursos asignados para la formación doctoral, superando la meta establecida de 500 doctores formados, incrementándose a 686, lo mismo que los servicios científicos 
y tecnológicos, cuya inversión pasó de USD 191602000 en 2010 a USD 213079000 en 2011. La administración y otras actividades de apoyo subieron la inversión de USD 45649000 en 2010 hasta USD 54097000 en 2011. Por último, se observa que las actividades de innovación tuvieron una menor inversión en 2011; se expone que en el 2010 se invirtieron USD 526198000 y en 2011 USD520164000. En 2010 se generó en este sentido una inversión general de USD 1403939000, mientras que en 2011 esta inversión alcanzó el monto de USD 1466970000 (Salazar et al., 2011). El CPC (2012) explica que, si bien existen diversas entidades financiadoras en Colombia de las ACTI, asimismo se observa la desarticulación entre los diferentes actores del ecosistema y la fragmentación de recursos; dicha fragmentación también genera poca utilización de estos recursos por parte de las empresas para la generación de innovación.

Tabla 3. Inversión nacional en ACTI 2010-2014 en dólares estadounidenses en Colombia Table 3. Colombia's national investment in STIA from 2010 to 2014 in US dollars

\begin{tabular}{lccccc}
\hline \multicolumn{1}{c}{ Tipo de actividad } & 2010 & 2011 & 2012 & 2013 & 2014 \\
\hline $\begin{array}{l}\text { Investigación y Desarrollo } \\
\begin{array}{l}\text { Formación y capacitación } \\
\text { científica y tecnológica }\end{array}\end{array}$ & 542352000 & 559885000 & 743722000 & 818686000 & 602969000 \\
\hline $\begin{array}{l}\text { Servicios científicos y } \\
\text { tecnológicos }\end{array}$ & 191602000 & 213079000 & 252707000 & 351213000 & 321042000 \\
\hline $\begin{array}{l}\text { Administración y otras } \\
\text { actividades de apoyo }\end{array}$ & 45649000 & 54097000 & 81867000 & 78093000 & 74952000 \\
\hline
\end{tabular}

Fuente: Salazar et al., (2011), Lucio et al., (2013) y Lucio et al., (2015).

Revisando la inversión nacional en ACTI en los años 2012 y 2013 en USD, se observa que la actividad investigación y desarrollo fue la más apoyada, invirtiendo, en el año 2012, la suma de USD 743722000 y en el 2013 el monto de USD 818686000. Las actividades de formación y capacitación científica y tecnológica también evidenciaron un incremento, subiendo de USD 128491000 en 2012 a USD 126530000 en 2013. Cabe resaltar que, según el Informe de Gestión 2012 (Colciencias, 2012), el número de personal beneficiario de doctorados aumentó en un $23 \%$ con relación al año anterior. Se otorgaron un total de 844 becas, de las cuales 440 se asignaron para programas doctorales en IES en Colombia y 404 en el exterior (Colciencias, 2012). En el año 2013 este apoyo se modificó, incrementando a 516 los cupos asignados para formación nacional en doctorados, mientras que para doctorados en el exterior se asignaron 141 becas (Colciencias, 2014). La inversión en servicios científicos y tecnológicos también evidenció un aumento, pasando de USD 252707000 en 2012 a USD 351213000 en 2013. La administración y otras actividades de apoyo igualmente evidencian más inversión con base al bienio anterior; en el año 2012 se reportó USD 81867000 y en el año 2013 bajó levemente a USD 78093000. Por último, las actividades de innovación tuvieron una menor inversión en este bienio (2012-2013); esto puede estar relacionado con la baja de inversión privada durante ese periodo. El monto asignado para estas actividades fue de USD 464603000 en 2012 y bajó a USD 450998000 en 2013. En un informe presentado por el DNP y la DSEPP (2013), se expone que hasta el año 2013 (equivalente al tercer año de gobierno del expresidente Juan Manuel Santos) se habían apoyado a 780 empresas en sus estrategias de innovación.

En este sentido, en 2012 se generó una inversión general de USD 1671392000, mientras que en 2013 esta inversión alcanzó el monto de USD 1825320000 (Lucio et al., 2013). Cabe agregar que en un informe generado por el CPC (2013) se expresa que Colombia necesitaba trabajar urgente una estrategia de innovación y contar con una entidad que asumiera el liderazgo del tema, con el fin de 
poder dinamizar los resultados de esta actividad. Por último, en un dictamen presentado por el DNP y la DSEPP (2014) se expone que, al cierre del año 2013, se habían aprobado 224 proyectos de regalías por valor de USD 882278000 para ser financiados a través de los recursos disponibles en el Fondo de Ciencia, Tecnología e Innovación del SGR.

Al revisar el discriminado de la inversión nacional en ACTI en USD, se puede evidenciar que para el año 2014 la actividad investigación y desarrollo tuvo una inversión de USD 602969000. Para este mismo año, la inversión en formación y capacitación científica y tecnológica fue de USD 79317000. Según el Informe de Gestión 2015 (Colciencias, 2015), de las 983 becas asignadas para estudios doctorales, 725 fueron para programas en universidades colombianas y 258 para estudios en IES en el extranjero. Un programa liderado por Colciencias, denominado «Es Tiempo de Volver», desarrollado en el 2014, apoyó el retorno de 123 investigadores colombianos con doctorado obtenido en el exterior para vincularse a 28 entidades en Colombia entre universidades, centros de investigación, gobierno, etc., (Colciencias, 2015). La inversión en servicios científicos y tecnológicos evidenció un incremento, reportando en 2014 una inversión de USD 321042000. Ese año, la administración y otras actividades de apoyo contaron con una inversión de USD 74952000. Igualmente se evidenció más inversión en las actividades de innovación, la cual fue de USD 454.289.000; se debe tener en cuenta que la inversión en innovación no volvió a ser tan alta durante los años estudiados, como en 2010, la cual fue de USD 526198000. Entre los años 2010 y 2014 se apoyaron a 1416 empresas con instrumentos de innovación; esto generó un cumplimiento de la meta establecida por el gobierno en un 105.7\% (Departamento Nacional de Planeación-DNP \& Dirección de Seguimiento y Evaluación de Políticas Públicas-DSEPP, 2015). Asimismo, en 2014 se generó una inversión general de USD 1532570000 (Lucio et al., 2015).

\section{Caso Ecuador: inversión en ACTI entre los años 2010-2014}

A continuación, se realizó una descripción anual de la inversión destinada a actividades de CTI en Ecuador desde el año 2010 hasta el 2014, conforme a los datos reportados por la Senescyt a través de la Encuesta Nacional de Actividades de Ciencia, Tecnología e Innovación (ACTI) y los diferentes informes de resultados presentados de manera anual de acuerdo a mandato constitucional.

De acuerdo con el Código Orgánico de la Economía Social de los Conocimientos, Creatividad e Innovación, la ciencia y la tecnología son los pilares de una sociedad basada en el conocimiento, con una orientación hacia una economía social que impulse el uso de saberes y la creatividad en la solución de las diferentes problemáticas sociales (Asamblea Nacional República de Ecuador, 2016). En este sentido Ecuador, a partir del 2010, ha tratado de destinar recursos considerables al desarrollo de actividades de CTI (actividades sistemáticas relacionadas con la producción, promoción, difusión y aplicación de los conocimientos científicos y técnicos en todos los campos de la ciencia y la tecnología [OCDE, 2015]) dentro de su territorio, financiamiento que en su momento pudo sostenerse únicamente con fondos públicos dados los elevados precios que mantenía el petróleo en el mercado mundial durante el periodo estudiado.

Al revisar los datos correspondientes a la Encuesta ACTI de la Senescyt (2014), se observa que, en el caso de Ecuador, para el año 2010, la inversión en estas actividades fue equivalente al $1.84 \%$ del PIB y que este valor tuvo variaciones significativas durante los años 2011 y 2012 (1.56\% y 1.58\%) debido, principalmente, a problemas de índole político al interior del país; sin embargo, ese porcentaje volvió 
a situarse en entre $1.98 \%$ y $1.88 \%$ durante los años 2013 y 2014 gracias a que el barril del petróleo durante esos años se mantuvo en un valor superior a los USD100 (ver Tabla 4).

Tabla 4. Porcentaje del PIB aportado para ACTI y Porcentaje del PIB aportado para I+D 2010-2014 en Ecuador Table 4. Percentage of Ecuador's GDP devoted to STIA and R\&D from 2010 to 2014

\begin{tabular}{lcc}
\hline Año & Porcentaje PIB-Inversión en ACTI & Porcentaje PIB-Inversión en I+D \\
\hline 2010 & $1.84 \%$ & $0.41 \%$ \\
\hline 2011 & $1.56 \%$ & $0.35 \%$ \\
\hline 2012 & $1.58 \%$ & $0.33 \%$ \\
\hline 2013 & $1.98 \%$ & $0.38 \%$ \\
\hline 2014 & $1.88 \%$ & $0.44 \%$ \\
\hline \multicolumn{3}{c}{ Fuente: Senescyt (2014) }
\end{tabular}

En cuanto a las acciones enfocadas en investigación y desarrollo, se observa que, entre los años 2010 y 2014, Ecuador destinó en promedio un $0.38 \%$ de su PIB. Si se considera que el gasto en I+D alcanzó un máximo de $0.44 \%$ en el 2014 (Senescyt, 2020), siendo el 1\% del PIB el porcentaje recomendado por la Unesco (2016) para este indicador en países en desarrollo, es posible señalar que el país no ha logrado sostener un financiamiento progresivo que permita dinamizar las actividades de I+D.

Vale la pena resaltar, sin embargo, que un importante flujo de estos recursos fue destinado al financiamiento de proyectos de investigación a través de la modalidad de convocatorias abiertas en las diferentes áreas del conocimiento priorizadas a nivel nacional, llegando a destinar, en 2013, la cifra récord de USD 39.43 millones (Senescyt, 2020) (ver Figura 2).

Monto financiado en millones de USD

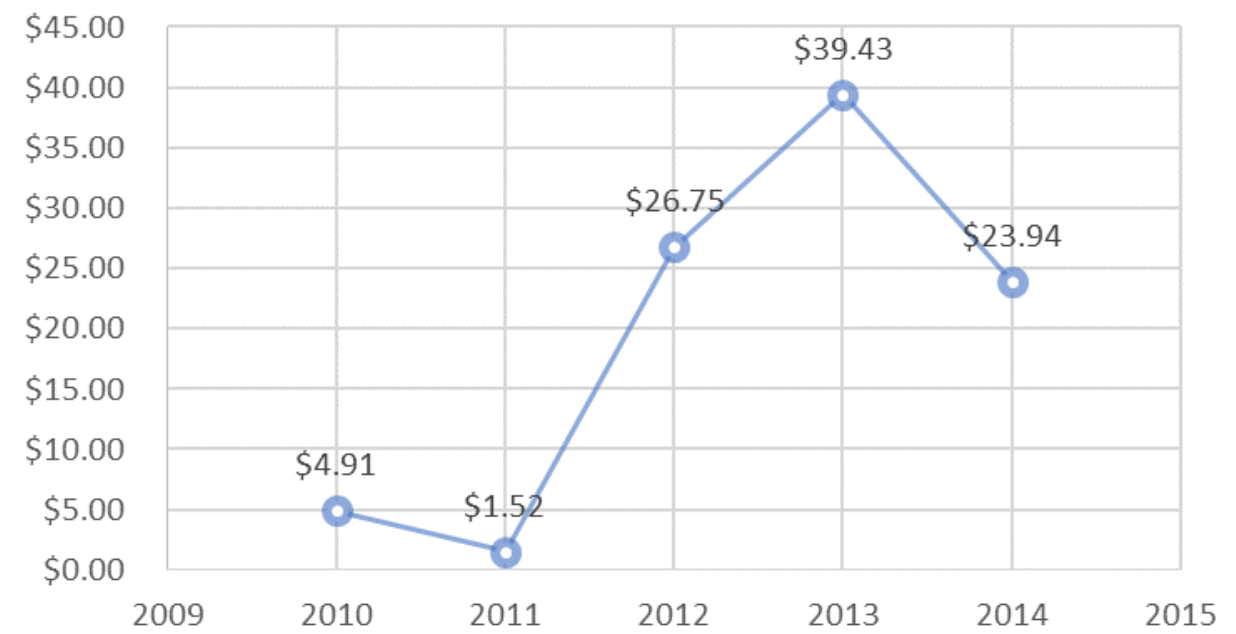

Figura 2. Financiamiento de la SENESCYT destinado a convocatorias abiertas, 2010 - 2014

Figure 2. SENESCYT funding of open calls from 2010 to 2014

Fuente: Senescyt (2014).

En lo pertinente las inversiones realizadas por tipo de actividades (ver Tabla 5), se observa que Ecuador, a partir del año 2010, invirtió en I+D USD 280.29 millones, cantidad que, pese a un 
decrecimiento menor en el año 2011 (USD 269.48 millones), en general muestra un crecimiento sostenido, llegando a invertir en el año 2014 la suma de USD 450.31 millones (Senescyt, 2020). Con respecto a actividades de ciencia y tecnología, de igual manera es posible apreciar un continuo crecimiento, iniciando en el año 2010 con USD 35.95 millones y terminando en el año 2014 con USD 123.11 millones, logrando un incremento del $342 \%$.

En lo relativo a actividades de innovación se pueden observar valores bastante altos, en particular porque aquí se incluyen aspectos como: «adquisición de maquinaria y equipo; adquisición de hardware; adquisición de software; adquisición de tecnología desincorporada; consultorías y asistencia técnica; ingeniería y diseño industrial; capacitación del personal; $y$, estudios de mercado» (OCDE, 2015). Tomando en cuenta lo mencionado, son notables las inversiones realizadas a partir del año 2010, en el cual se destinaron USD 927.75 millones en este tipo de actividades; para el año 2011 hubo un leve decrecimiento al llegar a invertirse USD 898.40 millones; sin embargo, a partir del 2012 se presenta un crecimiento constante durante tres años consecutivos, desde el 2012 al 2014, en los cuales se invirtieron USD 1017.76, USD 1361.14 y USD 1349.87 millones, respectivamente.

Tabla 5. Inversión nacional en ACTI 2010-2014 en millones de USD en Ecuador Table 5. Ecuador's national investment in STIA from 2010 to 2014 in millions of US dollars

\begin{tabular}{cccccc}
\hline Tipo de actividad & 2010 & 2011 & 2012 & 2013 & 2014 \\
\hline $\begin{array}{c}\text { Investigación y } \\
\text { desarrollo }\end{array}$ & 280.29 & 269.48 & 292.23 & 361.40 & 450.31 \\
\hline $\begin{array}{c}\text { Actividades de } \\
\text { ciencia y tecnología }\end{array}$ & 35.95 & 42.66 & 76.67 & 158.75 & 123.11 \\
\hline $\begin{array}{c}\text { Actividades de } \\
\text { innovación }\end{array}$ & 927.75 & 898.40 & 1017.76 & 1361.14 & 1349.87 \\
\hline
\end{tabular}

En Ecuador las IES y los institutos públicos de investigación (IPI) concentran la mayor parte de las actividades en I+D desarrolladas en el país (Senescyt, 2020). En el caso de las universidades, lo hacen desde un marco normativo que ubica a la investigación como una función sustantiva de su labor. El financiamiento a estas actividades proviene en gran parte de las asignaciones presupuestarias anuales públicas y se suman los fondos públicos de fomento a la I+D provenientes de la Senescyt, los cuales, en su mayoría, son destinados a la ejecución de proyectos de investigación y fortalecimiento de la gestión de investigación (ver Tabla 6).

Tabla 6. Gasto en I+D según sector de ejecución 2010-2014 en Ecuador Table 6. Ecuador's expenditure in R\&D between 2010 and 2014 classified by sector

\begin{tabular}{cccccc}
\hline Tipo de recurso & 2010 & 2011 & 2012 & 2013 & 2014 \\
\hline Públicos & 104.59 & 67.58 & 72.32 & 114.25 & 165.75 \\
\hline Educación Superior & 45.30 & 38.25 & 47.85 & 63.11 & 87.66 \\
\hline ONG & 8.75 & 7.03 & 4.75 & 6.74 & 6.40 \\
\hline & Fuente: Senescyt (2014).
\end{tabular}

Es importante mencionar que las IES también financian actividades de investigación a partir de fuentes de recursos alternativos a los públicos, es decir, distintos a los otorgados por Senescyt y los provenientes de su presupuesto de gasto corriente o inversión (Senescyt, 2020). Las IES en el país 
participan en convocatorias internacionales de financiamiento, al igual que generan compromisos en este ámbito para la gestión y obtención de fondos, entre otras iniciativas al respecto. Este documento no incluye esta información, debido a la disponibilidad; sin embargo, en el país se enfrentan grandes desafíos para gestionar este tipo de recursos. Por una parte, los desafíos están relacionados con el plazo de inclusión de estos fondos en el presupuesto de las IES, ya que se requiere de tiempos considerables para que puedan ser utilizados. Esto implica que las IES ecuatorianas se retrasen en la ejecución de las actividades de los proyectos en comparación con los centros de investigación de otros países, y que, por lo tanto, se incrementen las probabilidades de incumplir con los objetivos. En conclusión, el país pierde competitividad en esta área. Por otra parte, los desafíos están relacionados con el gasto de los fondos, es decir, respecto a la adquisición de equipos, insumos, bienes y servicios para la investigación. Las IES públicas se rigen por los procesos de contratación pública que afectan la celeridad de las compras, impactando, a su vez, la celeridad en el cumplimiento de las actividades de las investigaciones. De igual manera, el país pierde competitividad. Por estas razones, realizar investigación en las IES de Ecuador es complejo y requiere más tiempo del establecido para desarrollar esta actividad.

En función de los datos presentados, en términos generales se pueden observar los esfuerzos que Ecuador ha realizado (y que se encuentra realizando) con el objetivo de impulsar la ciencia, la tecnología y la innovación como herramientas que permitan generar una economía basada en el conocimiento y dejar de ser un país netamente dependiente de los ingresos producto de la exportación de petróleo; no obstante, resulta fundamental emprender una medición del impacto social, económico y científico del financiamiento realizado a estas actividades. El registro de este impacto puede derivar en herramientas de gran utilidad para visibilizar la importancia de la CTI para el desarrollo de un país, posicionar las políticas públicas dirigidas a este ámbito en la agenda política y definir intervenciones más efectivas. Al no contar con datos provenientes de las inversiones por parte del sector privado disponibles durante este periodo, es difícil establecer su involucramiento en las actividades de ciencia, tecnología e innovación.

\section{Resultados de las inversiones}

En este espacio se comparan el número becas y créditos asignados para maestría y doctorado, la producción bibliográfica, así como las patentes de invención y diseños industriales obtenidos por Ecuador y Colombia entre los años objeto de estudio (2010-2014). Estos elementos constituyen indicadores de resultados e impacto académico tal cual lo establecen manuales de CTI internacionales, como el Manual de Frascati y el Manual de Oslo, los cuales son referentes de medición de la inversión en CTI realizada por los países.

\section{Colombia}

En el periodo comprendido de 2010 a 2014, se observa que, en Colombia, solo en el año 2010, se asignaron 2941 becas/créditos de maestría y 731 de doctorado (ver Figura 3), observamos. Para el año 2011 hubo un incremento en estos apoyos, subiendo a 3239 en las becas/créditos de maestría y 836 de doctorado. En el 2012 continúa el incremento, siendo el año en donde se evidencia en productos de impacto un mayor resultado: 2826 becas/créditos para maestría y 1159 para doctorado. En los dos últimos años del periodo a revisar, las cifras bajan. En 2013, las becas/créditos asignados para maestría caen a 2130 y las de doctorado a 977. Finalmente, en el año 2014 se 
entregaron 2236 becas/créditos de maestrías, pero a su vez, se entregó el mayor número de becas de doctorado: 1492 (Lucio et al., 2015).

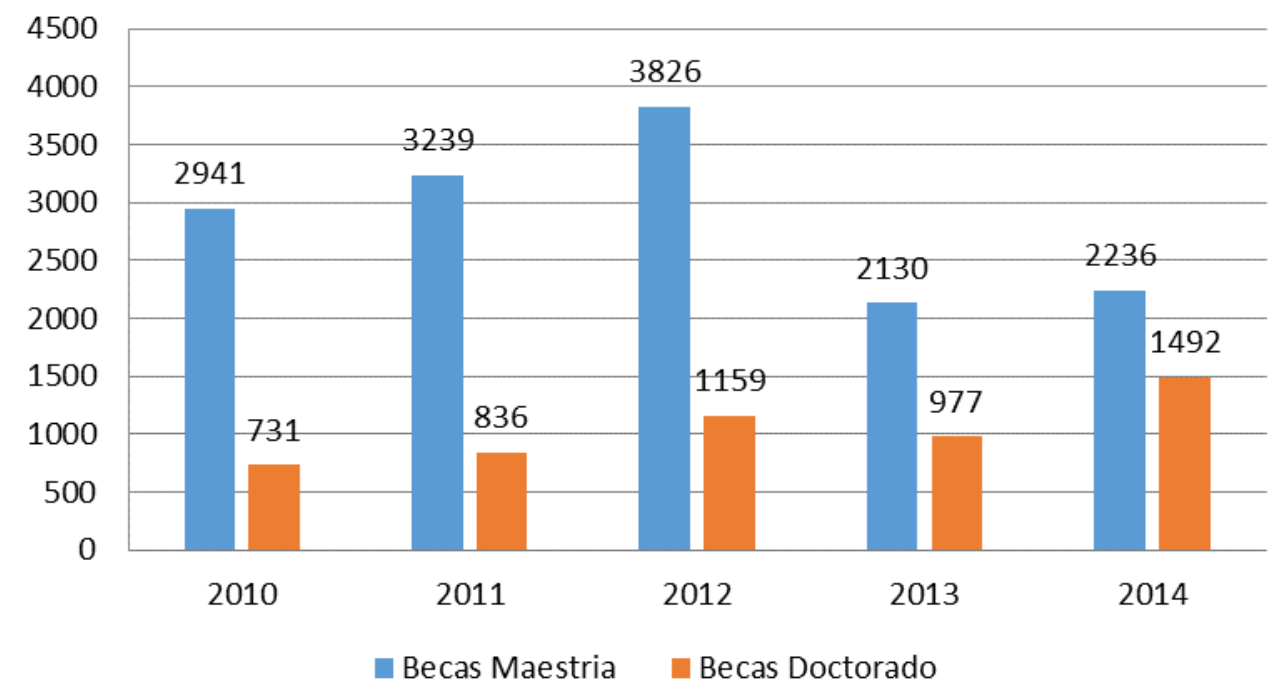

Figura 3. Becas y créditos asignados para programas de maestrías y doctorados en Colombia entre 2010-2014 Figure 3. Scholarships and loans for master's and doctoral programs granted by Colombia between 2010 and 2014

Fuente: Lucio et al., (2015).

Al revisar el tema de la producción bibliográfica en Colombia entre los años 2010-2014 (ver Tabla 7), como resultado de las inversiones realizadas en CTI, según la información reportada en la base de datos Web of Science, y del informe de Lucio-Arias et al., (2016), se observa que durante este periodo las cifras de los documentos y artículos registrados fueron aumento: en 2010 se registraron 5532 documentos y 6517 artículos; en 2011, el número de documentos incrementó a 6350 y el de artículos a 7295; en 2012, los documentos y artículos subieron a 6896 y 8048, respectivamente. En los años siguientes, la cifra siguió en aumento, identificando que, para el año 2013, 7008 documentos y 8175 artículos aparecían registrados en la plataforma Web of Science. Esto demuestra que la difusión de las investigaciones es un tema relevante, lo cual está relacionado con la cantidad de proyectos de investigación que se tengan en curso. También estos datos van muy de la mano con los criterios de autoevaluación y acreditación de programas, establecidos por el Ministerio de Educación Nacional (MEN) y el Consejo Nacional de Acreditación (CNA). Asimismo, como es sabido, para que los grupos de investigación y los investigadores en Colombia pudieran subir en las categorías establecidas por Colciencias, las publicaciones eran y siguen siendo uno de los indicadores más importantes.

Tabla 7. Producción bibliográfica de autores vinculados a instituciones colombianas en revistas indexadas en Web of Science 
Table 7. Bibliographic production of authors affiliated to Colombian institutions in journals indexed in Web of Science

\begin{tabular}{ccc}
\hline Año & Documentos & Artículos \\
\hline 2010 & 5532 & 6517 \\
\hline 2011 & 6350 & 7295 \\
\hline 2012 & 6896 & 8048 \\
\hline 2013 & 7008 & 8175 \\
\hline 2014 & 7136 & 8269 \\
\hline \multicolumn{3}{c}{ Fuente: Lucio-Arias et al., (2016). }
\end{tabular}

Al revisar el aspecto de las patentes asignadas por vía nacional en Colombia 2010-2014 (ver Tabla 8), se observa que: en el año 2010 se entregaron 21 títulos de patentes a residentes y 60 a no residentes; en 2011 se asignaron 31 patentes a residentes, frente a 45 a no residentes; para el 2012, 101 patentes fueron asignadas a residentes y 130 a no residentes. En los dos últimos años objeto de estudio la cifra no sufre cambios marcados: en 2013 se asignaron 136 a residentes y 107 a no residentes; finalmente, en 2014, se entregaron 110 a residentes y 65 a no residentes; esto demuestra la baja producción científica y el desconocimiento generalizado del sistema nacional de producción intelectual en Colombia.

Algo interesante para anotar es que entre el año 2010 y el 2014 se redujo sustancialmente el tiempo para el trámite de derechos de patentes en Colombia, pasando de 60.4 meses en 2010, a 24.3 meses en 2014 (DNP y DSEPP, 2015).

Tabla 8. Patentes asignadas por vía nacional

Table 8. Patents registered by residents and non-residents in Colombia

\begin{tabular}{ccc}
\hline Año & Patentes vía nacional asignadas \\
\hline & Residentes & Año \\
\hline 2010 & 21 & 2010 \\
\hline 2011 & 31 & 2011 \\
\hline 2012 & 101 & 2012 \\
\hline 2013 & 136 & 2013 \\
\hline 2014 & 110 & 2014 \\
\hline
\end{tabular}

Fuente: Lucio-Arias et al., (2016).

En cuanto a los diseños industriales (ver Tabla 9), se observa que, en Colombia, en el año 2010, se asignaron 69 patentes a residentes y 261 a no residentes. Para el 2011, el incremento llegó a 312 para residentes y 505 para no residentes. En 2012 se entregaron 191 para residentes y 279 para no residentes. En el año 2013, fueron 172 para residentes y 363 para no residentes. Por último, en 2014 la cifra fue de 209 para residentes y 313 para no residentes. Esto puede estar relacionado con un desconocimiento de los procedimientos correspondientes para el registro y la falta de una mayor articulación entre los actores de CTI, especialmente con las empresas.

Tabla 9. Diseños industriales concedidos en Colombia entre los años 2010-2014 
Table 9. Industrial designs registered by Colombia between 2010 and 2014

\begin{tabular}{ccc}
\hline Año & Residentes & No residentes \\
\hline 2010 & 69 & 261 \\
\hline 2011 & 312 & 505 \\
\hline 2012 & 191 & 279 \\
\hline 2013 & 172 & 363 \\
\hline 2014 & 209 & 313 \\
\hline \multicolumn{3}{c}{ Fuente: Lucio-Arias et al., (2016). }
\end{tabular}

\section{Ecuador}

En 2008 se expide la vigente Constitución de la República del Ecuador, en la cual se consagra la gratuidad de la educación hasta el tercer nivel. Desafortunadamente, en ese momento no se tomó en cuenta la carencia de docentes calificados para la enseñanza universitaria, lo que motivó a la implementación de una política de becas ambiciosa para estudios de posgrado en el extranjero con el objetivo de suplir la falta de docentes, bajo el supuesto de que las competencias y capacidades para la investigación científica y la producción de saber se desarrollan, en términos formales, a partir de los estudios de cuarto nivel. De igual manera, esta política de becas de estudios de cuarto nivel (maestrías y doctorados) en el extranjero, estuvo directamente relacionada a la idea de cambiar de matriz productiva nacional, la cual hasta el día de hoy se caracteriza por ser netamente primarioexportadora, contar con un déficit de mano de obra cualificada, la permanente salida de recursos humanos a países con mejores salarios, lo que permite recuperar la inversión realizada en educación, y debido a la falta de políticas públicas que permitan un acceso equitativo a una educación superior de calidad (Secretaría de Educación Superior, Ciencia, Tecnología e Innovación, 2014).

Con estos antecedentes es posible explicar el porqué de la gran inversión destinada a la profesionalización de talento humano en Ecuador y pasar de entregar 660 becas para estudios de cuarto nivel en el 2010 a 10482 becas en el año 2014 (ver Figura 4). Durante este periodo, Ecuador es el país de América Latina que entrega la mayor cantidad de becas al año con relación al tamaño de su población: 2.27 por cada 10000 habitantes, seguido por Brasil con 1.64; Chile 0.55; y México 0.13 (Senescyt, 2014).

Entre los múltiples criterios establecidos para evaluar y acreditar adecuadamente a las universidades (academia, eficiencia académica, investigación, organización e infraestructura), se incluye la categorización de su cuerpo docente, los cuales, entre los diferentes parámetros que deben considerar para bajar, mantenerse o subir de categoría, se encuentra precisamente la generación y el impacto logrado a través de su producción científica (Consejo de Evaluación, Acreditación y Aseguramiento de la Calidad de la Educación Superior [CEAACES], 2014).

Si se toma en cuenta que de la evaluación y acreditación obtenida por la IES dependía la asignación presupuestaria a recibir el año siguiente, y que, en el caso del personal docente, de acuerdo a su categorización, varía el salario a percibir, es lógico pensar el impulso que se dio a la generación de producción científica. 


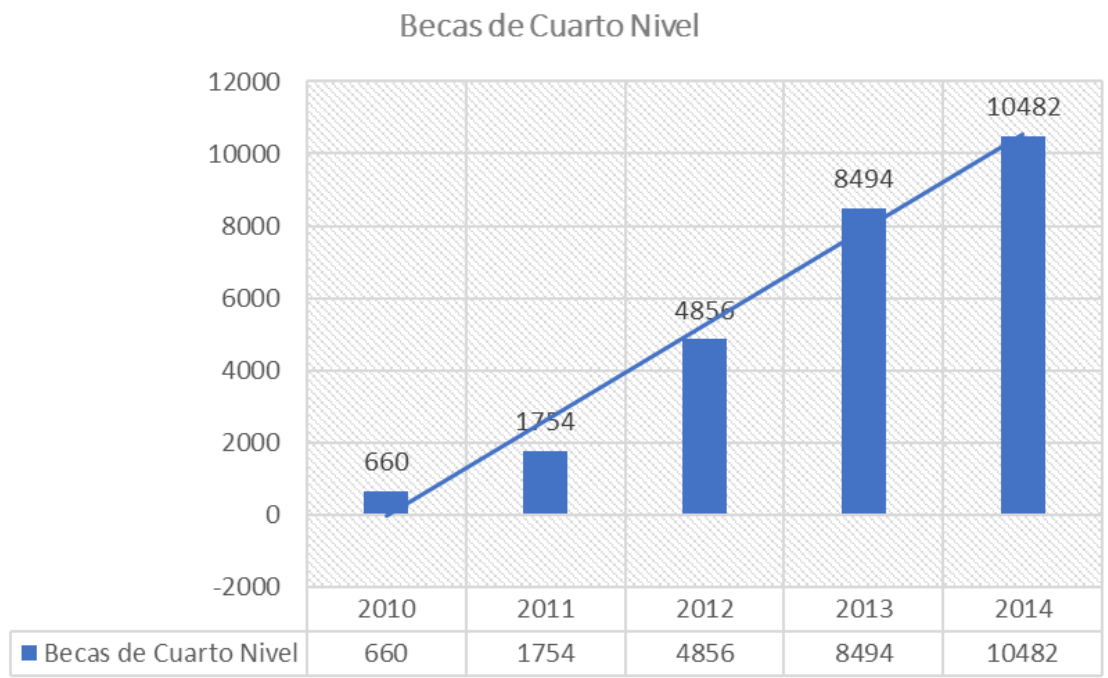

Figura 4. Becas de Cuarto Nivel entregadas en Ecuador entre 2010-2014

Figure 4. PhD scholarships granted by Ecuador between 2010 and 2014 Fuente: Senescyt (2014).

Con estos antecedentes se puede observar que, en Ecuador, entre los años 2010-2014 (ver Tabla 10), como resultado de las políticas públicas implementadas, y gracias a la inversión realizada en CTI, las publicaciones indexadas crecieron de manera sostenida, pasando de 452 en el 2010, hasta llegar a 1006 publicaciones en el 2014, esto de acuerdo a la información reportada en la base de datos Web of Science.

Tabla 10. Producción bibliográfica ecuatoriana en revistas indexadas durante los años 2010-2014 de acuerdo con el portal Web of Science

Table 10. Bibliographic production of authors affiliated to Ecuadorian institutions in journals indexed in Web of Science between 2010 and 2014

\begin{tabular}{cc}
\hline Año & Publicaciones indexadas \\
\hline 2010 & 452 \\
\hline 2011 & 485 \\
\hline 2012 & 642 \\
\hline 2013 & 741 \\
\hline 2014 & 1006 \\
\hline & Fuente: Senescyt (2014).
\end{tabular}

El entorno de innovación de un país se conoce en virtud de los elementos citados a continuación: «regulaciones, control de calidad, propiedad intelectual, normalización y metrología, acceso al financiamiento, incentivos fiscales, sistema impositivo y las TIC» (Schwartz \& Guaipatín, 2014). El tema de propiedad intelectual incorpora principalmente los aspectos relativos a patentes de invención y diseños industriales.

Lastimosamente, en virtud de las políticas públicas implementadas en todo el Sistema Nacional de Ciencia, Tecnología e Innovación, a partir de la entrada en vigencia de la Ley Orgánica de Educación Superior del año 2010, la cual prioriza la producción científica sobre la propiedad intelectual, es 
posible observar el decrecimiento en el número entre los años 2010 y 2014, registrándose 105 patentes de invención para residentes en el 2010 y llegando a apenas un número de 20 en el 2014. En el caso de patentes de invención para no residentes el número no varía de manera tan drástica, ya que estos no se veían influenciados por la mencionada ley 2014 (ver Tabla 11).

Tabla 11. Patentes asignadas en Ecuador por vía nacional

Table 11. Patents registered by residents and non-residents in Ecuador

\begin{tabular}{ccc}
\hline Año & Residentes & No residentes \\
\hline 2010 & 105 & 563 \\
\hline 2011 & 66 & 548 \\
\hline 2012 & 82 & 468 \\
\hline 2013 & 24 & 358 \\
\hline 2014 & 20 & 475 \\
\hline Fuente: Servicio Nacional de Derechos Intelectuales (s. f.).
\end{tabular}

Para el caso de los diseños industriales concedidos, estos no se vieron afectados por una ley que se encontraba netamente enfocada en la comunidad académica, razón por la cual los números, durante el periodo 2010-2014, mantuvieron su promedio, tanto para residentes como no residentes, conforme se muestra en la parte inferior (ver Tabla 12).

Tabla 12. Diseños industriales concedidos en Ecuador entre los años 2010-2014

Table 12. Industrial designs registered by Ecuador between 2010 and 2014

\begin{tabular}{ccc}
\hline Año & Residentes & No residentes \\
\hline 2010 & 52 & 70 \\
\hline 2011 & 55 & 77 \\
\hline 2012 & 82 & 118 \\
\hline 2013 & 80 & 70 \\
\hline 2014 & 65 & 95 \\
\hline
\end{tabular}

Fuente: Servicio Nacional de Derechos Intelectuales (s. f.).

\section{DISCUSIÓN}

Durante el periodo estudiado (2010-2014), Colombia y Ecuador aún no alcanzaban las recomendaciones expuestas por la Unesco (2016) frente a la inversión en I+D, la cual debería equivaler al $1 \%$ del PIB, según lo establecido por este organismo para países en vía de desarrollo, alcanzando, en 2014, el $0.251 \%$ en Colombia y el 0.44\%. en Ecuador. Bortagaray (2016) indica que la inversión en las actividades de ciencia, tecnología e innovación en América Latina por lo general provienen del sector público y esto se evidencia claramente tanto en el caso colombiano como en el ecuatoriano. Si bien en Colombia el $44.25 \%$ de los recursos para las ACTI en 2014 fueron otorgados por el sector privado, en Ecuador estos datos no están disponibles para el periodo de estudio. En este sentido se evidencia para ambos países durante esta etapa, la necesidad de una mayor inversión por parte de los sectores productivos para las ACTI, así como por parte de otros sectores, como la sociedad civil organizada y entidades internacionales. 
Si bien en Latinoamérica en el periodo estudiando había un déficit de capital humano, lo cual tuvo como resultado que aún no se contase con una masa crítica relevante para el desarrollo de los sistemas de CTI en la región (Crespi \& Dutrénit, 2013), se observan importantes inversiones por parte de Colombia y Ecuador para la formación de capital humano, evidenciando, en el caso de Colombia, la asignación de un total de becas para maestría y doctorado de 19567, mientras que en el caso de Ecuador, siendo un país más pequeño, la inversión fue mayor, al evidenciarse la asignación de 26246 becas, tal y como se expuso en el apartado de resultados. Cabe resaltar que la inversión en capital humano se da especialmente para la formación de futuros investigadores, sin embargo, se observa que se deben fortalecer las inversiones que se realizan en educación científica desde edades tempranas en los colegios, con el fin de fortalecer competencias como la indagación y el pensamiento científico, tal y como lo expresa Ibañez-Marti (2018). Siguiendo a Narro Robles (2008), en Colombia y Ecuador aún se debe continuar fortaleciendo y generando una masa crítica que pueda desarrollar estas competencias relacionadas con la innovación y el pensamiento crítico y científico.

Según la Unesco (2015), las patentes otorgadas en América Latina representaron alrededor del 1\% del número concedido a las economías de altos ingresos, según datos reportados del año 2013. En el caso de Colombia y Ecuador se evidencia una gran diferencia en el registro de patentes dado que, en el periodo estudiado, en Colombia solo se registraron un total de 806, frente a 2709 registradas en Ecuador. En el caso de los diseños industriales, se observa por parte de Colombia un total de 2674 concedidos, mientras que a Ecuador se le asignaron 764. Por otra parte, la cantidad de artículos publicados en revistas de impacto creció sustancialmente, especialmente en países como Colombia, el cual registra un total de 38304 artículos publicados en Web of Science, frente a 3326 reportados en Ecuador.

Cabe resaltar que las inversiones en CTI deberán acompañarse, según los autores (Pérez Hernández et al., 2018), de medidas y políticas sociales que permitan que la población vulnerable de los países objeto de estudio se vinculen a actividades de CTI. Durante el periodo observado, estas políticas no han sido evidentes del material estudiado. Por otra parte, y siguiendo a García Guzmán (2011), las políticas e inversiones de CTI deben tener una proyección a largo plazo, lo cual no es evidente al revisar las fluctuaciones en las inversiones en ambos países en los años revisados. En este sentido, estos estudios son relevantes para examinar a detalle las inversiones en CTI a través de los años y reflexionar sobre los programas, inversiones y políticas realizadas por los gobiernos, así como el impacto que han tenido las mismas en el tiempo. También son importantes para compararlos con la situación actual de inversión, así como las prioridades y la proyección en la materia hacia el futuro. Por último, estos estudios comparados sirven para evidenciar tendencias en inversiones entre los países y revisar proyectos y programas que se consideren buenas prácticas y puedan ser adaptados a los propios contextos.

\section{CONCLUSIONES}

Más allá de los recursos asignados en los cinco años estudiados, se evidenció que la inversión efectuada en Colombia durante este periodo, si bien impactó publicaciones y la formación de alto nivel, el registro de patentes pudo mejorar. En el caso de Ecuador resaltan la gran inversión en la formación de alto nivel de capital humano y el número importante de patentes registradas en el periodo objeto de estudio; un aspecto en el que se pudo mejorar fueron los indicadores de las 
publicaciones en bases de datos de impacto. Lastimosamente, durante el período estudiado no es claro, en el caso de Ecuador, cuáles han sido los recursos asignados por parte del sector productivo. De todos modos, la inversión por parte del sector productivo se ha evidenciado como un reto en los esquemas de CTI colombiano durante el periodo estudiado. También llama la atención la baja gestión de recursos internacionales para CTI.

Si bien se observan importantes inversiones en la formación de capital humano y futuros investigadores, se requiere contar con esfuerzos que puedan apoyar al desarrollo del pensamiento científico desde los niños en las escuelas, para empezar a generar masa crítica con competencias hacia la indagación y la innovación.

No está claro cómo estas inversiones se articulan con programas que sirvan a población vulnerable y fomenten su inclusión en la CTI y el desarrollo de conocimiento. Asimismo, es necesario generar estrategias e inversiones a largo plazo que puedan darle continuidad a los esfuerzos y de esta manera alcanzar objetivos que puedan tener impacto en el desarrollo y la competitividad de los países. En este orden de ideas, se hacen necesarios estudios de impacto para las iniciativas y programas de CTI de los países, con el fin de medir si las inversiones realizadas han generado el impacto esperado entre los actores involucrados y en el cumplimiento de las metas de gobierno.

\section{CONFLICTOS DE INTERÉS}

Los autores declaran que no presentan conflictos de interés financiero, profesional o personal que pueda influir de forma inapropiada en los resultados obtenidos o las interpretaciones propuestas.

\section{CONTRIBUCIÓN DE AUTORES}

Para el desarrollo de este proyecto todos los autores han realizado una contribución significativa, especificada a continuación:

Luisa Fernanda Echeverría-King: trabajó la inversión de Colombia en las ACTI y la metodología, así como realizó contribuciones a los apartados de resultados de las inversiones, discusión, resumen y conclusiones.

Jorge Pinto: realizó el apartado correspondiente a la inversión de Ecuador en las ACTI, así como realizó contribuciones a los apartados de resultados de las inversiones, discusión y conclusiones.

María Ángela Lorena Mosquera-Montoya: trabajó introducción y marco teórico, así como realizó contribuciones a los apartados de la discusión y conclusiones.

\section{REFERENCIAS}

Abello, R.; Páez, J.; Dacunha, C. (2001). ¿Son la ciencia y la tecnología un instrumento de desarrollo? Un análisis de caso para américa latina. Investigación y Desarrollo, v. 9, n. 1, 372-387. URL 
Aguirre-Bastos, C.; Gupta, M. P. (2009). Science, technology and innovation policies in Latin America: Do they work? Interciencia, v. 34, n. 12, 865-872. URL

Albert, M.; Laberge, S. (2007). The legitimation and dissemination processes of the innovation system approach: The case of the Canadian and Québec science and technology policy. Science, Technology, \& Human Values, v. 32, n. 2, 221-249. https://doi.org/10.1177/0162243906296854

Baquero Méndez, D.; Mieles López, J. D. (2015, 8 de abril). Los booms petroleros: ¿Qué cambió en los últimos 40 años? Foro Economía Ecuador. URL

Bortagaray, I. (2016). Políticas de Ciencia, Tecnología, e Innovación sustentable e Inclusiva en América Latina. UNESCO. URL

Casas, R. (2019). Políticas públicas de ciencia y tecnología en América Latina. Ante la encrucijada de los cambios políticos. Teuken Bidikay, v. 11, n. 16, 21-28. https://doi.org/10.33571/teuken.v11n16a1

Chaves, C. V.; Ribeiro, L. C.; Dos Santos, U. P.; Albuquerque, E. M. (2020). Sistemas de innovación y cambios en la división centro-periferia: notas sobre una metodología para determinar las trayectorias de los países a partir de las estadísticas de ciencia y tecnología. Revista CEPAL, $\mathrm{n}$. 130, 45-64. URL

Cheng, H.; Wang, B. (2019). Multiplier effect of science and technology innovation in regional economic development: Based on panel data of coastal cities. Journal of Coastal Research, v. 94, n. sup. 1, 883-890. https://doi.org/10.2112/S194-175.1

Código Orgánico de la Economía Social de los Conocimientos, creatividad e innovación. (2016, 9 de diciembre). Asamblea Nacional República de Ecuador. Registro Oficial No. 899. URL

Consejo Privado de Competitividad de Colombia. (2011). Informe Nacional de Competitividad 20102011: Ruta a la prosperidad colectiva. URL

Consejo Privado de Competitividad de Colombia. (2012). Informe Nacional de Competitividad 20112012: Ruta a la prosperidad colectiva. URL

Consejo Privado de Competitividad de Colombia. (2013). Informe Nacional de Competitividad 20122013: Ruta a la prosperidad colectiva. URL

Constitución Política de Colombia. (1991) Artículo 71 (Colombia).

Constitución Política de Ecuador. (2008) Artículo 277 y 298 (Ecuador).

Crespi, G.; Dutrénit, G. (Eds.). (2013). Políticas de ciencia, tecnología e innovación para el desarrollo: La experiencia latinoamericana. Foro Consultivo Científico y Tecnológico. URL 
Decreto 585 de 1991. Por el cual se crea el consejo nacional de ciencia y tecnología, se reorganiza el instituto colombiano para el desarrollo de la ciencia y la tecnología -Colciencias-y se dictan otras disposiciones. (1991, 26 de febrero). Presidencia de la República. D. O. No. 39702.

Departamento Administrativo de Ciencia, Tecnología e Innovación. (2011). Informe de gestión 2011. $\underline{U R L}$

Departamento Administrativo de Ciencia, Tecnología e Innovación. (2012). Informe de Gestión 2012. $\underline{U R L}$

Departamento Administrativo de Ciencia, Tecnología e Innovación. (2014). Informe de gestión institucional 2013. URL

Departamento Administrativo de Ciencia, Tecnología e Innovación. (2015). Informe de gestión 2015. $\underline{U R L}$

Departamento Administrativo Nacional de Estadística. (2014). Educación Superior presentó la mayor inversión en Actividades Científicas, Tecnológicas y de Innovación en el bienio 2012-2013. URL

Departamento Nacional de Planeación. (2014). Bases del Plan Nacional de Desarrollo 2014-2018. URL

Departamento Nacional de Planeación-DNP; Dirección de Seguimiento y Evaluación de Políticas Públicas-DSEPP. (2012). Informe al Congreso. Juan Manuel Santos 2012. URL

Departamento Nacional de Planeación-DNP; Dirección de Seguimiento y Evaluación de Políticas Públicas-DSEPP. (2013). Informe al Congreso. Juan Manuel Santos 2013. URL

Departamento Nacional de Planeación-DNP; Dirección de Seguimiento y Evaluación de Políticas Públicas-DSEPP. (2015). Informe al Congreso. Juan Manuel Santos 2015. URL

Etokakpan, M. U.; Adedoyin, F. F.; Vedat, Y.; Bekun, F. V. (2020). Does globalization in Turkey induce increased energy consumption: insights into its environmental pros and cons. Environmental Science and Pollution Research, v. 27, 26125-26140. https://doi.org/10.1007/s11356-020-08714-3

Franco Avellaneda, M.; Linsingen, I. (2011). Polarizaciones de la ciencia y la tecnología en América Latina. Mirando la política científica en clave educativa. Revista Mexicana de Investigación Educativa, v. 16, n. 51, 1253-1272. URL

García Guzmán, M. (2011). Políticas de innovación científica y tecnológica en América Latina. Encrucijada. Revista Electrónica Del Centro De Estudios En Administración Pública, n. 7, 1-12. https://doi.org/10.22201/fcpys.20071949e.2011.7.58487

Giraldo Gutiérrez, F. L.; Ortiz Clavijo, L. F.; Zúñiga-Miranda, S. (2020). Políticas de Ciencia, Tecnología e Innovación en América Latina y el Caribe y su influencia en la producción y apropiación de la CTI. Linguagem e Ensino, v. 23, n. 1, 292-316. https://doi.org/10.15210/rle.v23i1.17751 
Gómez Londoño, R. (2015, 23 de noviembre). Colombia no supo administrar la bonanza petrolera. Sectorial. URL

Ibañez-Marti, J. J. (2018). La Ciencia en Latinoamérica: Tendencias y patrones. Revista de la Facultad de Ciencias, v. 7, n. 1, 23-39. https://doi.org/10.15446/rev.fac.cienc.v7n1.69409

Lee, C.; Park, G.; Kang, J. (2018). The impact of convergence between science and technology on innovation. The Journal of Technology Transfer, v. 43, n. 2, 522-544. https://doi.org/10.1007/s10961-016-9480-9

Lemus Delgado, D. R. (2020). Vietnam: políticas públicas en ciencia, tecnología e innovación. Estudios de Asia y África, v. 55, n. 2, 263-294. https://doi.org/10.24201/eaa.v55i2.2454

Ley 1286 de 2009. Por la cual se modifica la ley 29 de 1990, se transforma Colciencias en Departamento Administrativo, se fortalece el sistema de Ciencia, Tecnología e Innovación en Colombia y se dictan otras disposiciones. (2009, 23 de enero). Congreso de la República. D. O. No. 47241.

Ley 1530 de 2012. Por la cual se regula la organización y el funcionamiento del Sistema General de Regalías. (2012, 17 de mayo). Congreso de la República. D. O. No. 48433.

Loray, R. (2017). Políticas públicas en ciencia, tecnología e innovación: tendencias regionales y espacios de convergencia. Revista de Estudios Sociales, n. 62, 68-80. https://doi.org/10.7440/res62.2017.07

Lucio, J.; Lucio-Arias, D.; Rivera Torres, S. C.; Tique, J.; Villareal, N. F.; Villarreal, N. F.; Lozano-Borda, M.; Daza-Caicedo, S.; Mora, H.; Perea, G. I.; Bueno, E.; Guevera, A.; Salinas Pico, J. M.; CárdenasOsorio, J. F.; Galvis-Restrepo, M.; Aguilar, X.; Torralba Barreto, D. R.; Barón, V.; Cruz, D. A. (2013). Indicadores de Ciencia y Tecnología Colombia 2013. Edición de Bolsillo. Bogotá: Observatorio Colombiano de Ciencia y Tecnología. URL

Lucio, J.; Rivera Torres, S. C.; Tique Ortiz, J.; Lucio-Arias, D.; Mora Holguín, H.; Pardo Martínez, C. I.; Guevera, A.; Perea, G. I.; Torralba Barreto, D. R.; Garavito Muñoz, M. P.; Melo Martínez, F. H.; Márques-Bustos, N.; Zárate Rincón, S. M.; Castro Novoa, N. (2015). Indicadores de Ciencia y Tecnología Colombia 2015. Observatorio Colombiano de Ciencia y Tecnología. URL

Lucio-Arias, D.; Rivera Torres, S. C.; Tique Ortiz, J.; Villareal, N. F.; Lucio J.; Mora, H.; Perea, G. I.; Guevara, A.; Salinas Pico, J. M.; Torralba Barreto, D. R.; Melo Martinez, F. H.; Márquez, N.; Garavito Muñoz, M. P. (2016). Indicadores de Ciencia y Tecnología Colombia 2016. Edición de Bolsillo. Observatorio Colombiano de Ciencia y Tecnología. URL

Maravert Alba, M. I.; Molina Hernández, J. A.; Molina Ramírez, J. A. (2016). El Gasto En Investigación Y Desarrollo Experimental (GIDE) en México, promotor del crecimiento económico. Ciencia Administrativa, n. 1, 109-125. $\underline{\text { URL }}$ 
Martín Marín, B. (2011). Investigación descriptiva. En Cubo Delgado, S; Martín Marín, B.; Ramos Sánchez, J. L. (Coords.), Métodos de investigación y análisis de datos en ciencias sociales y de la salud (373-386). Ediciones Pirámide.

Narro Robles, J. (2008). Educación, ciencia y desarrollo: El caso de América Latina. Perfiles educativos, v. 30 , n. $119,90-103 . \underline{U R L}$

Organización de las Naciones Unidas para la Educación, la Ciencia y la Cultura. (2016). ¿Cuánto invierten los países en I+D? Una nueva herramienta de la UNESCO identifica a los nuevos protagonistas. Servicio de Prensa. $\underline{\mathrm{URL}}$

Organización de las Naciones Unidas para la Educación, la Ciencia y la Cultura. (2015). UNESCO science report: towards 2030. UNESCO Publishing. URL

Organización para la Cooperación y el Desarrollo Económicos. (2015). Frascati Manual 2015: Guidelines for Collecting and Reporting Data on Research and Experimental Development, The Measurement of Scientific, Technological and Innovation Activities. OCDE. https://doi.org/10.1787/9789264239012-en

Pérez Hernández, C. C.; Gómez Hernández, D.; Lara Gómez, G. (2018). Determinantes de la capacidad tecnológica en América Latina: una aplicación empírica con datos de panel. Economía: teoría y práctica, n. 48, 75-123. https://doi.org/10.24275/etypuam/ne/482018/perez

Perfetti, J. J. (2010). Ciencia, tecnología e innovación (CT+I). En Steiner, R.; Traverso, V. (Eds.), Colombia 2010-2014: propuestas de política pública (321-374). Fedesarrollo y CAF. http://hdl.handle.net/11445/2916

Red de Indicadores de Ciencia y Tecnología -Iberoamericana e Interamericana (2020). El Estado de la Ciencia. Principales Indicadores de Ciencia y Tecnología Iberoamericanos / Interamericanos 2020. UNESCO y OEI. URL

Resolución No. 110-CEAACES-S0-13-2014. Reglamento para los procesos de autoevaluación de las instituciones, carreras y programas del sistema de Educación Superior. (2014). Consejo de Evaluación, Acreditación y Aseguramiento de la Calidad de la Educación Superior. URL

Salazar, M.; Lucio, J.; Rivera, S. C.; Bernal, E.; Ruiz, C.; Usgame, C.; Lucio-Arias, D.; Daza Caicedo, S.; Guerrero, J.; Guevara, A.; Perea, G. I.; Cifuentes, F.; García, M.; Pérez, S.; Sanchez, E. (2011). Indicadores de Ciencia y Tecnología Colombia 2011. Observatorio Colombiano de Ciencia y Tecnología. URL

Scargiali, E. A. (2019). Las políticas de Estado en Ciencia y Tecnología en el marco de los procesos de integración regional en América Latina. Revista electrónica de estudios latinoamericanos, v. 18, n. 71, 37-50. URL 
Schwartz, L.; Guaipatín, C. (2014). Ecuador. Análisis del Sistema Nacional de Innovación: Hacia la consolidación de una cultura innovadora. Banco Interamericano de Desarrollo. $\underline{\mathrm{UR}}$

Secretaría de Educación Superior, Ciencia, Tecnología e Innovación. (2014). Principales Indicadores de Actividades de Ciencia, Tecnología e Innovación. Instituto Nacional de Estadísticas y Censos.

Secretaría de Educación Superior, Ciencia, Tecnología e Innovación. (2020). Principales Indicadores de Ciencia, Tecnología e Innovación. Instituto Nacional de Estadísticas y Censos.

Servicio Nacional de Derechos Intelectuales. (s.f). Diseños industriales concedidos en Ecuador entre los años 2010-2014. URL

Velo, A. B.; Perrotta, D. V. (2020). La cooperación científica y tecnológica entre el MERCOSUR y la Unión Europea. Revista de la Secretaría del Tribunal Permanente de Revisión, v. 8, n. 16, 117144. https://doi.org/10.16890/rstpr.a8.n16.p117

Wu, M.; Zhao, M.; Wu, Z. (2019). Evaluation of development level and economic contribution ratio of science and technology innovation in eastern China. Technology in Society, v. 59, 101194. https://doi.org/10.1016/j.techsoc.2019.101194 